\title{
Diagnostic performance of volume and limited oblique MRI of the anterior cruciate ligament compared to knee arthroscopy
}

\author{
Paula J. Richards ${ }^{1}$ \\ lain McCall ${ }^{1}$ \\ Alexandra Kraus ${ }^{1}$ \\ Mary Jones ${ }^{2}$ \\ Gayle Maffulli ${ }^{3}$ \\ Stephen Bridgman ${ }^{3}$ \\ Nicola Maffulli ${ }^{4}$ \\ 1 Department of Radiological Sciences, University \\ Hospital of North Staffordshire NHS Trust, Stoke on \\ Trent, UK \\ 2 School of Computing and Mathematics, University \\ of Keele, UK \\ ${ }^{3}$ Orthopaedic Surgical Trials Unit, Department of \\ Trauma and Orthopaedic Surgery, Keele University \\ School of Medicine, Guy Hilton Research Centre, \\ Thornburrow Drive, Hartshill, Stoke on Trent, UK \\ ${ }^{4}$ Department of Musculoskeletal Disorders \\ Faculty of Medicine, Surgery and Dentistry, \\ University of Salerno, Salerno, Italy; \\ Centre for Sports and Exercise Medicine \\ Barts and London School of Medicine and Dentistry, \\ Queen Mary University of London, London, UK
}

Corresponding author:

Nicola Maffulli

Department of Musculoskeletal Disorders

Faculty of Medicine, Surgery and Dentistry

University of Salerno, Salerno, Italy

E-mail: n.maffulli@qmul.ac.uk

\section{Summary}

Background: Many strategies have been used to improve the visualisation of the $A C L$ including sagittal, coronal oblique sequences, and $3 D$ volume imaging. Nevertheless, the ACL may not always be visualised.

Methods: Two hundred and thirty-one consecutive patients (77 females; 154 males; average age 43.5, range 18 to 82 years; 205 with chronic, 20 acute, and 6 acute on chronic symptoms) underwent knee arthroscopy for mechanical symptoms within a week of MRI. After routine orthogonal sequences, if general MRI radiographers, with over four years experience, were not able to identify the presence of the ACL, then two 3D volume sequences and 2D limited sagittal oblique T1 sequences were per- formed. Patients requiring extra sequences, missed by the radiography technicians, were recalled. The MRI sequences were evaluated in a blinded fashion by three radiologists, and compared to the knee arthroscopy findings, with the normal ACL acting as internal controls. The radiography technicians performed additional ACL sequences in 63 patients $(27 \%)$; of these, 10 patients had a partial and 12 patients had a complete ACL tear. Only 2 patients $(0.6 \%)$ were recalled (one with a normal, and one with a full thickness ACL tear).

Results: The filmed ACL evaluation for complete tears and a normal ACL had a sensitivity of $100 \%$, specificity of $97.1 \%$ and accuracy of $97.3 \%$, slightly higher than evaluating on the monitor. Volume sequences had specificities and accuracies over $95 \%$, with good intraobserver reliability (Kappa $\mathbf{0 . 8 5 9}$, $95 \% \mathrm{Cl}$ 0.705-1.0). Experienced radiographers identified most cases requiring supplementary MRI ACL sequences. An additional volume sequence was beneficial when filmed. Use of the monitor can offer some benefits. Limited oblique $\mathrm{T} 1$ sequence of the intercondylar notch was unreliable.

KEY WORDS: ACL MRI, anterior cruciate ligament, knee arthroscopy, MRI, volume sequences.

\section{Introduction}

Clinical evaluation for anterior cruciate ligament $(A C L)$ is good in isolation, but more difficult in chronic symptoms and is not as reliable in traumatic haemarthrosis and acute locking ${ }^{1}$, whilst knee arthroscopy is not without risk ${ }^{2}$. MRI is valuable for diagnosis, allows appropriate surgical planning, with previously unsuspected diagnoses in $33 \%$ and a change in management in $63 \%$ of patients ${ }^{3}$. MRI has a high diagnostic performance for complete ACL ruptures ${ }^{4}$. Many strategies have been used to improve the visualisation of the ACL including sagittal, coronal oblique sequences, and 3D volume imaging ${ }^{5-8}$. Nevertheless, the ACL may not always be visualised ${ }^{9-12}$ either for technical reasons or because it is ruptured and absent. Partial tears are not obviously difficult to identify ${ }^{13-16}$, especially when chronic ${ }^{16}$, on conventional MRI sequences. We hypothesised that general $\mathrm{MRI}$ radiographers with over four years experience could select abnormal ACLs and apply supplementary sequences appropriately. It would be wasteful to apply supplementary sequences to all patients and 
the literature has not compared the additional sequences available. We assessed the radiographers ability to apply further imaging. We compared, in a double blind fashion, the diagnostic performance of two volume sequences (DESS and FLASH) and a limited oblique thin section $\mathrm{T} 1$ of the intercondylar notch of the ACL with arthroscopy.

\section{Method}

All procedures were performed according to what described in Padulo et al. ${ }^{17}$. A previous study evaluating the impact of MRI on the rate of knee arthroscopy performed for mechanical knee symptoms from 4.6.01 to 30.9.02 had a cohort of 231 patients who had knee MRI and arthroscopy within a week ${ }^{18}$. They received standard clinical management ${ }^{18}$, with those patients with a normal ACL acting as internal controls. The study received approval by the Local Ethics Committee, and all patients gave written informed consent ${ }^{18}$. Patients were excluded if an MRI or an arthroscopy of the affected knee had been performed within the past 12 months, if they had MRI contraindications, previous ACL surgery, or if the patient did not undergo arthroscopy ${ }^{18}$. Patients with a possible tumour, synovial disease, arthropathy or multiple trauma, including dislocation of the knee or the patella, were also excluded ${ }^{18}$. The three radiologists (a radiology specialist registrar, and two musculoskeletal radiologists of five and over 20 years experience) were blind to each other's evaluations, to the patients' notes, the trial data, and did not assess the patients clinically. The findings of the three radiologists were compared to the findings at arthroscopy, which was considered as the gold standard for the diagnosis of a torn ACL. No events which might have changed the diagnosis were recorded in the week between MRI and arthroscopy relating to the knee.

All MR studies were performed using a 1T Magnetom Impact Expert (Siemens Ltd, Siemens House, Bracknell, Oldbury, RG12 8FZ) with the dedicated knee coil. The studies were imaged on films, and recorded and saved on optical discs for subsequent evaluation on the monitor. Each patient underwent an axial localiser, sagittal T1 and gradient echo T2*, coronal STIR, and axial fat suppressed dual echo, and subsequently the data were reviewed in relation to the MRI evaluation of the $A C L$. If no normal ACL was identified by the radiographer, additional sagittal sequences (3D DESS and 3D fat suppressed GE flash and limited oblique T1 of the intercondylar notch) were performed, or the patients were immediately recalled after review by one musculoskeletal radiology consultants. The six general MRI radiographers each had over four years experience of prescribing MRI sequences and identifying the normal appearance of the ACL on routine sequences. The radiographers made and filmed the double oblique reconstructions of the ACL. The parameters for all the sequences are given in Table I. Each MRI sequence was evaluated by one musculoskeletal radiologist and, if the routine sequences of printed images did not reveal the $A C L$, then the $3 D$ DESS raw data on the monitor were evaluated with double oblique reconstructions. A second musculoskeletal radiologist three years later independently evaluated only the volume sequences filmed and, on the monitor, the double oblique reconstructions (DESS $1.6 \mathrm{~mm}$ and FLASH $2 \mathrm{~mm}$ ), without knowledge of the findings on the other sequences and using those patients with normal ACL as internal controls. The two radiologists independently evaluated the conventional filmed oblique 2D T1 sequences without any other sequences, all blinded to the previous evaluation, and to the results of arthroscopy, 18 months after the initial analysis, again using the normal ACL as internal controls.

The criteria to diagnose a complete tear of the ACL were loss of continuity of the ACL with a relatively horizontal position, bowing or "hyperbuckled" posterior cruciate ligament $4,8,9,19$, and absence of the ACL. A partial tear was diagnosed if there was abnormal intra-substance signal with some discernable ligament fibres, injury to only one ACL bundle, bowing or undulating contour of the ACL but with some preserved fibres.

The ACL was classified as normal if a continuous low signal ligament was seen on the sagittal and coronal sequences parallel to the intercondylar line. The re-

Table I. MRI sequence parameters.

\begin{tabular}{|c|c|c|c|c|c|c|c|c|c|}
\hline Sequences & FOV & Matrix & TR & TE & FA & NEC & TI & Time & $\begin{array}{l}\text { Thickness } \\
(\mathrm{mm})\end{array}$ \\
\hline Sagittal T1 & 200 & $256 \times 256$ & 532 & 15 & $90^{\circ}$ & 2 & - & $2 \mathrm{~m} 28 \mathrm{~s}$ & 4 \\
\hline Sagittal T2* & 200 & $241 \times 256$ & 608 & 18 & $20^{\circ}$ & 2 & - & $4 \mathrm{~m} 55 \mathrm{~s}$ & 4 \\
\hline Axial FS DE & 220 & $190 \times 256$ & 3500 & $22 / 90$ & $80^{\circ}$ & 2 & - & $4 \mathrm{~m} 29 \mathrm{~s}$ & 5 \\
\hline Coronal STIR & 210 & $196 \times 256$ & 4300 & 30 & $180^{\circ}$ & 1 & 120 & $4 m 6 s$ & 4 \\
\hline Sagittal FS3D & 180 & $168 \times 256$ & 58 & 11 & $40^{\circ}$ & 1 & - & $7 \mathrm{~m} 30 \mathrm{~s}$ & 2 \\
\hline Sagittal DESS & 200 & $256 \times 256$ & 26.8 & 9 & $40^{\circ}$ & 1 & - & $4 \mathrm{~m} 49 \mathrm{~s}$ & 1.6 \\
\hline Oblique T1 & 220 & $224 \times 256$ & 400 & 15 & $90^{\circ}$ & 3 & - & $2 \mathrm{~m} 27 \mathrm{~s}$ & 3 \\
\hline
\end{tabular}

Key: FOV: Field of view; TR: Time to Repetition; TE: Time to Excitation; FA:Flip angle; NEC: Acquisitions; TI: Time to inversion. 
sults were compared to the arthroscopy findings. Variances between the radiologists were reviewed, and a consensus reached.

\section{Statistics}

The intra- and inter-observer variations were calculated, and the kappa statistic determined. Sensitivity and specificity, positive and negative predictive values were calculated for each strategy. Reliability was measured using the Kappa statistics. This chancecorrected index of agreement has a value of zero when agreement is no better than chance, and a maximum of one when agreement is perfect. Interpretation was according to Shrout's classification: a value of Kappa between 0 and 0.1 representing virtually no agreement, 0.11-0.4 slight, 0.41-0.6 fair, 0.61-0.8 moderate, and $0.81-1$ substantial agreement.

\section{Results}

All 231 patients had both MRI and arthroscopy ${ }^{18}$. There were 154 males and 77 females, with an average age of 43.5 years (range 18 to 82 ). Most had chronic symptoms (205 patients), and the remaining had acute (20 patients) or acute on chronic (6 patients) symptoms. There were no posterior cruciate, medial collateral or lateral collateral ligament tears in the cohort. There were 22 (12 complete and 10 partial) ACL tears at arthroscopy. One hundred and sixty-eight of 231 (73\%) patients were diagnosed as having a normal ACL based on the judgement of the radiography technicians at the time of scanning using the conventional MRI sequences, were normal at arthroscopy, and no further imaging was performed. Sixty-three of 231 patients $(27 \%)$, two of whom were not initially selected by the radiographers, had further $A C L$ sequences, and were recalled for further $A C L$ sequences following review by the first radiology consultant. The overall recall rate was $0.6 \%(2 / 231)$ : one patient had a normal knee at arthroscopy, and the other had a complete ACL tear.

For the whole study, using all filmed sequences and including both partial and complete tears as a single category of ACL tear, the overall sensitivity was $86.4 \%$, specificity was $95.2 \%$, and accuracy was $94.7 \%$ (Tab. II). The results, when using the monitor, were 63.6, 95.7 and $93 \%$ respectively (Tab. II). The mis-characterisation of partial tears as complete and vice-versa adversely affected the diagnostic performance statistics, even though, when an ACL lesion was present, the ACL was correctly diagnosed as abnormal. If partial ACL tears were excluded, then the sensitivity examining the actual films was $100 \%$, specificity was $97.1 \%$, and accuracy was $97.3 \%$.

\section{Evaluation of filmed limited oblique T1 of the in- tercondylar notch alone}

Complete data were available on 61 of the 63 patients who had additional sequences, which included
21 of the $22 \mathrm{ACL}$ tears. The two radiologists reported the ACL as torn or normal (Tab. III). The agreement between evaluators was slight (Tab. IV), with a Kappa of 0.42 , and a wide $95 \%$ confidence interval of 0.211 to 0.628 . The number of patients is relatively small, but the results suggest that oblique films have reasonable sensitivity but poor specificity, reasonable inter-observer agreement, a low positive and high negative predictive value.

\section{Comparison of two different volume methods}

Comparisons of FLASH and DESS sequences were performed in the 37 of the 63 subjects in whom the raw data discs allowed evaluation on the monitor. The agreement between the volume methods evaluated on the monitor was good, with a Kappa of 0.859 , and a $95 \%$ confidence interval of 0.705 to 1.00 . The numbers are very small, because the original scanner was decommissioned, and there were technical problems with the optical discs, nevertheless there was good agreement between the two routine methods.

\section{Discussion}

The technical performance of MRI at locating the ACL is high, when oblique sagittal slices at $20^{\circ}$ (range 10$30^{\circ}$ ) to the lateral femoral condyle 20,21 show the ACL throughout its length $(35 \times 10 \mathrm{~mm})$ as a homogenous black signal structure, running antero-medially (Fig. 1). Templates to prescribe the angle accurately ${ }^{22}$ or supplementary sagittal 6 or coronal oblique images ${ }^{6,11}$ may help in the evaluation of the ACL. This study shows that when the ACL is clearly visible as a low signal band parallel to the intercondylar line and on the coronal view as two bundles in a fan shape or an inverted ' $\mathrm{V}$ ', extending from the tibial plateau to the inner aspect of the lateral femoral condyle in the intercondylar notch, it is normal at arthroscopy, and can be reliably identified by radiography technicians. In this case, additional sequences can be avoided in most MR examinations for mechanical knee symptoms.

MRI has a high reported diagnostic performance for complete ACL tears, with pooled data reporting a sensitivity of $89 \%(95 \% \mathrm{Cl} 0.86-0.92)$, specificity of $94 \%(0.93-0.96)$ and accuracy of $93 \%(0.02-0.94)^{3}$, with the primary signs more sensitive than secondary signs $^{8}$. ACL diagnosis hinges on being able to see the ligament, but, because the ligament is not straight, it is difficult to visualise it along its length. Therefore, externally rotating the foot, and thus rotating the lower limb, is a way to try and help straighten the ligament. Although Polly et al. ${ }^{23}$ reported a very high accuracy of $97.3 \%$, actually they excluded the ACLs which they could not see, which meant that, for technical reasons, some may not have been visible, but some of them would have actually been ruptured; hence, as many were excluded on this basis, their reported accuracy is questionable. Although our study showed a relatively high sensitivity for complete $\mathrm{ACL}$ 
Table II a. Evaluation of the filmed sequences (including volume sequences).

\begin{tabular}{|c|c|c|c|c|c|}
\hline \multirow[b]{2}{*}{ Rater 1} & \multicolumn{3}{|c|}{ Arthroscopy } & \multirow[b]{2}{*}{ Total } & \\
\hline & Partial & Complete & Normal & & \\
\hline Partial & 1 & 0 & 0 & 1 & \\
\hline Complete & 6 & 12 & 0 & 28 & \\
\hline \multirow[t]{2}{*}{ Normal } & 3 & 0 & 199 & 202 & \\
\hline & 10 & 12 & 209 & 231 & \\
\hline Rater 1 & Tear & & Normal & & \\
\hline Tear & 19 & & 10 & 29 & \\
\hline \multirow[t]{2}{*}{ Normal } & 3 & & 199 & 202 & \\
\hline & 22 & & 209 & 231 & \\
\hline Rater & Sensitivity & & $95 \% \mathrm{Cl}$ & Specificity & $95 \% \mathrm{Cl}$ \\
\hline 2 & 0.727 & & $0.518-0.868$ & 0.919 & $0.874-0.949$ \\
\hline 1 & 0.864 & & $0.667-0.953$ & 0.952 & $0.914-0.974$ \\
\hline
\end{tabular}

Table II b. Evaluation of sequences on the monitor.

\begin{tabular}{|c|c|c|c|c|c|}
\hline \multirow[b]{2}{*}{ Rater 1} & \multicolumn{3}{|c|}{ Arthroscopy } & \multirow[b]{2}{*}{ Total } & \\
\hline & Partial & Complete & Normal & & \\
\hline Partial & 1 & 0 & 3 & 4 & \\
\hline Complete & 3 & 10 & 6 & 19 & \\
\hline \multirow[t]{3}{*}{ Normal } & 6 & 2 & 200 & 208 & \\
\hline & 10 & 12 & 209 & 231 & \\
\hline & \multicolumn{3}{|c|}{ Arthroscopy } & & \\
\hline Rater 1 & Partial & & Normal & Total & \\
\hline Partial tear & 14 & & 9 & 23 & \\
\hline \multirow[t]{2}{*}{ Normal } & 8 & & 200 & 208 & \\
\hline & 22 & & 209 & 231 & \\
\hline Rater & Sensitivity & & $95 \% \mathrm{Cl}$ & Specificity & $95 \% \mathrm{Cl}$ \\
\hline 2 & 0.556 & & $0.337-0.754$ & 0.976 & $0.945-0.990$ \\
\hline 1 & 0.636 & & $0.430-0.803$ & 0.957 & $0.920-0.977$ \\
\hline
\end{tabular}

tears using the limited oblique T1 Spin Echo (SE) sequence, the lower specificity resulted from non-visualisation of a small number of intact $A C L$ at

Table III. Limited intercondylar notch oblique T1 weighted Spin Echo sequence compared to Arthroscopy for two readers (The film for one $A C L$ tear and one normal ACL were missing, hence the total is 61 not 63 ).

\begin{tabular}{llllllll}
\hline $\begin{array}{l}\text { Limited } \\
\text { Oblique T1 }\end{array}$ & \multicolumn{3}{l}{ Arthroscopy } & \multicolumn{2}{l}{ Total } \\
\hline \multicolumn{3}{l}{ Tear } & \multicolumn{2}{c}{ Normal } \\
\hline Rater & 1 & 3 & 1 & 3 & 1 & 3 \\
\hline Tear & 20 & 19 & 26 & 19 & 46 & 38 \\
Normal & 1 & 2 & 14 & 21 & 15 & 23 \\
\hline Total & 21 & & 40 & & 61 &
\end{tabular}

arthroscopy. Poor visualisation of the normal ACL is a recognised problem in $5-10 \% 22$ and up to $22 \%$ of patients $^{23}$, and in these patients extra sequences are likely to be of benefit. Barry et al. evaluated the different imaging patterns of ACL injury with non-visualisation as the main pattern in $18 \%$ of knees $^{24}$, with the lowest positive predictive value $(82 \%)$ ascribed to poor positioning and volume averaging of the ligament (4-5 mm T1 and T2 SE). However, absence of an identifiable ACL in general correlated well with surgically confirmed ACL tears, and the $3 D$ sequence was accurate in the identification of a normal ACL. In our study, non-visualisation on the $3 \mathrm{D}$ reconstruction produced six false positives, possibly consequent to the loss of fat signal on the sequences used, reducing the contrast. The spatial resolution of coronal STIR sequences is also less than a SE or GE T2. One of our "false positive" patients from non-visualisation underwent ACL reconstruction four months lat- 


\section{P. J.Richards et al.}

Table IV. Inter-observer variation in reading limited oblique T1 weighted sequence.

\begin{tabular}{|c|c|c|c|c|}
\hline Rater & & 3 & & 1 \\
\hline Sensitivity $(95 \% \mathrm{Cl})$ & 0.905 & $(0.771-0.973)$ & 0.952 & (0.773-0.992) \\
\hline Specificity $(95 \% \mathrm{Cl})$ & 0.525 & $(0.375-0.671)$ & 0.350 & $(0.221-0.505)$ \\
\hline PPV (95\% Cl) & 0.500 & $(0.348-0.652)$ & 0.435 & $(0.302-0.578)$ \\
\hline NPV (95\% Cl) & 0.913 & $(0.732-0.976)$ & 0.933 & $(0.702-0.988)$ \\
\hline
\end{tabular}
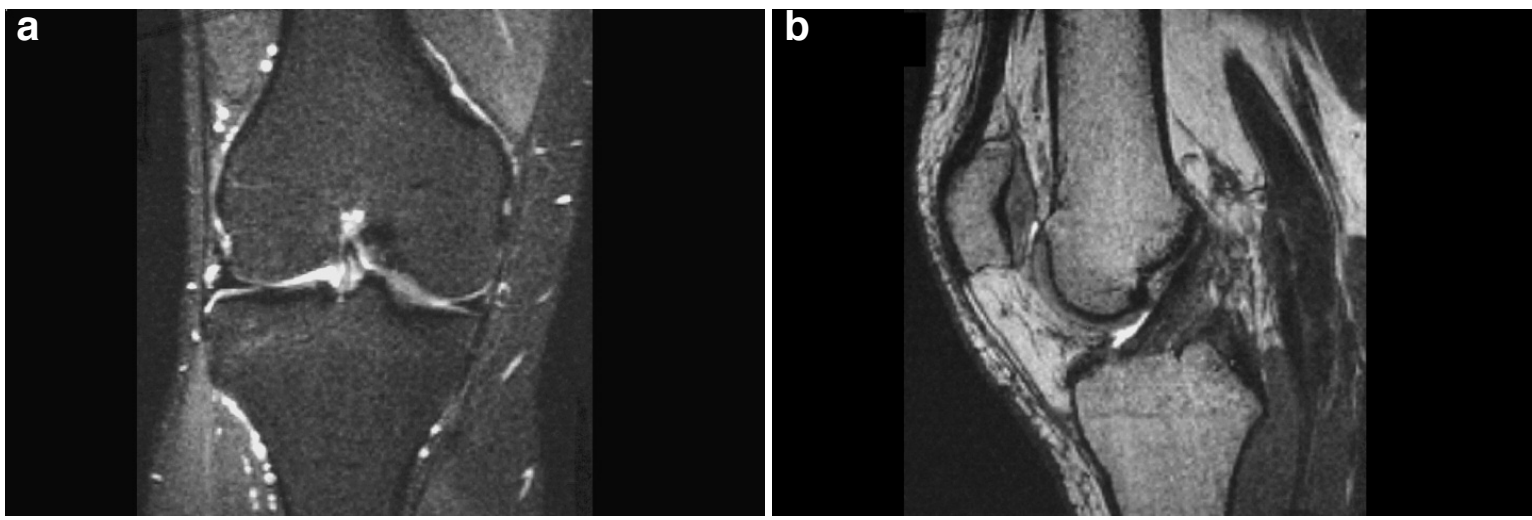

Figure 1. Normal appearance of the ACL on DESS.

a) Coronal STIR shows the typical inverted 'V' appearance of the ACL (black arrows) surrounded by a small amount of fluid.

b) Sagittal DESS shows the fluid as bright white line on T2 weighting (black arrow), but T1 shows normal anatomy, so the $\mathrm{ACL}$ is of low signal and intact. The cartilage visualised as different to the fluid.

er, possibly a false negative arthroscopy. However, at review, we noted a diffuse increase in signal and enlargement, possibly contusion or haemorrhage without a complete tear. Alternatively, degeneration or macroscopic twisting of the diverging bundles or ligament degeneration in our older cohort may contribute to non visualisation ${ }^{25}$.

The accuracy of MRI in chronic ACL tears is lower than in acute ones ${ }^{26}$. Hence, volume sequences, producing ultra-thin slices with reduced partial volume effect increase the sensitivity, specificity, accuracy and positive predictive value ${ }^{8,27}$. Reeder et al., using absence of an identifiable ACL, ACL laxity or discontinuity and bowing or hyperflexion of the posterior cruciate ligament as criteria, found the sensitivity and specificity of T1SE $3 \mathrm{~mm}$ interleaved sections to be 82 and $95 \%$ respectively ${ }^{8}$. A 3D Fast Imaging with Steady State Precession (FISP) sequence with 1.5 $\mathrm{mm}$ sections inexplicably reduced the sensitivity to $64 \%$, but increased specificity to $100 \%$, and they recommended using both sequences. Although counter intuitive, it is akin to our finding that the double oblique reconstructions made from the $3 \mathrm{D}$ volume had a higher diagnostic impact when reviewed printed on film than on the monitor. This is of particular relevance now that filmless systems predominate. Mori et al. ${ }^{7}$ used 3D GE $1.5 \mathrm{~mm}$ slices (0.5T) on potential chronic partial ACL compared to arthroscopy.
A partial tear was correctly diagnosed in $81.3 \%$ $(13 / 16)$, and complete tear in $92.9 \%(65 / 70)$ of chronic tears. Heron et al. ${ }^{28}$ used 3D FESS sagittal and coronal, that is $Z$, planes in 100 patients, with 20 complete and 3 partial tears at arthroscopy, 3 false positive and 2 false negative complete tears, and one true positive, one false positive and one false negative partial tears. Fitzgerald et al. $^{6}$ reported that improved accuracy of the ACL diagnosis by volume three orthogonal planes and obliques, but the present study has not shown any improvement in accuracy when reconstructing the $A C L$ from one $3 D$ sequence on the monitor. Our patients with limited oblique T1 sequences had a reasonable sensitivity but poor specificity, positive predictive value and intra-observer agreement.

The diagnosis of partial ACL tears in our patients was low, as reported in the literature ${ }^{12-15,29-31}$ (Fig. 2). In our cohort, six of the 10 partial ACL tears were diagnosed as complete. Chen et al. ${ }^{13}$, using $2 \mathrm{D}$ sagittal, coronal T1 and T2SE in chronic ACL tears, showed that a straight and tight ACL fibre implied a partial rather than complete tear. An empty notch or a wavy ACL were usually complete tears. In acute injuries, a focal increase in signal within the ACL favoured a diagnosis of a partial tear ${ }^{13}$. Umans et al., in a selected group, found that sensitivity for partial tears with different readers ranged from 40 to $75 \%$, and specificity 

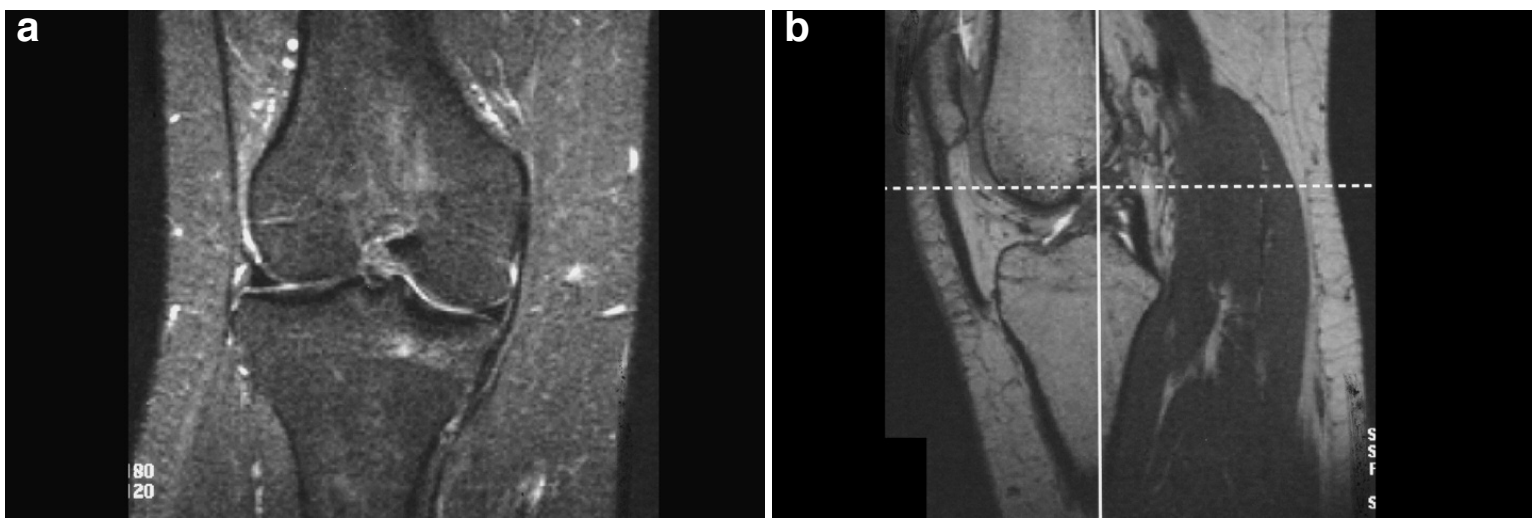

Figure 2. Arthroscopy showed a partial tear of the ACL.

a) Coronal STIR shows high signal in a component of the anteromedial bundle of the ACL. b) Sagittal double oblique DESS showed only one ACL bundle.

from 62 to $89 \%^{16}$. Lawrence et al. reported only $11 \%$ agreement between MR and arthroscopy for partial tears using volume sequences ${ }^{15}$.

The diagnosis of partial ACL tear is confounded by their relative rarity and lack of consensus regarding arthroscopic definition, as in one of our false negative complete ACL tears on MRI appeared to be intact and oedematous on consensus review (Fig. 3). There may be a group of patients with partial ACL tears at arthroscopy who have an apparently intact $A C L$ on MR because of the synovial covering (commonly names ligamentum mucosum) which also appears firm on probing at arthroscopy. Fast compared to conventional spin echo sequences, at $2 \mathrm{~T}$, show an improvement in diagnosing partial, but not complete, $\mathrm{ACL}$ tears $^{32,33}$, but these very high field $2 \mathrm{~T}$ magnets are expensive and not widely in use.

Our hypothesis that the thin slices and reduced volume averaging of volume sequences would improve the performance statistics over limited oblique T1 sequence is confirmed. The volume sequences allowed these $1.6 \mathrm{~mm}$ DESS or $2 \mathrm{~mm}$ FLASH reconstructions, whilst the oblique $\mathrm{T} 1$ slices could only be performed at $3 \mathrm{~mm}$, so some of our differences may arise from the slice thickness, not the sequence. However, our results do not confirm the additional benefit from making double oblique reconstructions on the monitor.

The limitations of the present study include the relatively small number tears, particularly partial ACL tears, with selection bias of using only the cohort who the radiographers thought abnormal being evaluated by all the MRI supplementary sequences. To truly evaluate the sequences against arthroscopy, all patients should have had all the sequence, but time and financial constraints prevented this. Our cohort was composed of older patients with predominantly chronic mechanical symptoms, which may also explain the relatively high number of partial tears ${ }^{18}$. To determine the diagnostic performance of an MRI sequence of the ACL, ideally would need injured ligaments, not a cohort with mechanical symptoms as the ones we studied. The small number of tears precluded formal

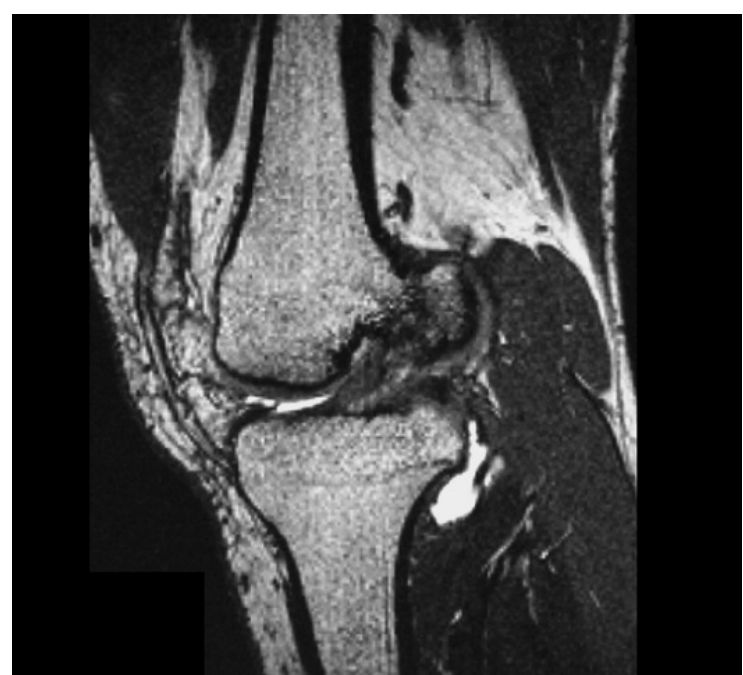

Figure 3. Arthroscopy showed a complete tear. On imaging, the ACL was oedematous but intact on both $3 \mathrm{D}$, but rather horizontal. The limited oblique $\mathrm{T} 1$ of the ACL was diagnosed as a complete ACL tear.

further analysis of individual features, and are reflected in the wide confidence intervals. The small number of tears meant that the radiology consultants may have remembered the findings and lead to the long intervals between each evaluation. Unfortunately, when the MRI scanner was decommissioned, rereading the optical discs on the later model proved unexpectedly problematic. The study would have benefited from all the radiologists undertaking all parts of the evaluation.

In conclusion, this study formally evaluated the interobserver reliability of assessing the ACL with double oblique reconstructions for a DESS, FLASH 3D volume, or limited oblique thin section T1's, compared to arthroscopy. The addition of a volume sequence by the unsupervised $M R I$ radiographer was helpful in evaluation of the ACL, and did not lead to over scan- 
ning with few recalls. It did not matter which volume sequence was used, as the diagnostic performance of both was high, with good inter-observer reliability, but limited oblique T1 was not reliable. Experienced radiographers identified most cases requiring supplementary MRI ACL sequences. An additional volume sequence was beneficial when filmed, but limited oblique T1 sequence of the intercondylar notch is unreliable.

\section{Acknoweledgements}

We wish to acknowledge our colleagues at the University Hospital of North Staffordshire NHS Trust and Keele University who have participated in the study; the Consultant Orthopaedic Surgeons Mr. David Griffiths, Mr. El Nasri Ahmed, and Mr. Ian dos Remedios. In particular, we wish to thank the staff of the MRI department, and Mrs. Cynthia Jackson for the preparation of this manuscript.

\section{Conflict of interests}

The Authors have no financial involvement and have no conflicts of interest.

\section{References}

1. McNally EG, Nasser KN, Dawson S, Goh LA. Role of magnetic resonance imaging in the clinical management of the acutely locked knee. Skeletal Radiology. 2002;31:570-573.

2. Sherman OH, Fox JM, Snyder SJ, et al. Arthroscopy - "NoProblem Surgery". J Bone Joint Surg (Am). 1986;68:256-265.

3. Mackenzie R, Palmer CR, Lomas DJ, Dixon AK. MRI of the knee: Diagnostic Performance Statistics. Clin Rad. 1996;51: 251-257.

4. Boeree NR, Ackroyd CE. Magnetic Resonance Imaging of Anterior Cruciate Ligament Rupture. J Bone Joint Surg (Br). 1992;74B:614-616.

5. Breitenseher MJ, Mayerhoefer ME. Oblique MR Imaging of the Anterior Cruciate Ligament Based on Three-Dimensional Orientation. J Magnetic Res Imaging. 2007;26:794-798.

6. Fitzgerald SW, Remer EM, Friedman H, Rogers LF, Hendrix RW, Schafer MF. MR Evaluation of the Anterior Cruciate Ligament: Value of Supplementing Sagittal Images with Coronal and Axial Images. AJR. 1993;160:1233-1237.

7. Mori A, Shirai Y, Nakayama Y, Narita T. Three-dimensional gradient-echo MR imaging of chronic partial anterior cruciate ligament tears. J Nippon Med Sch. 1998;65:5:429-431.

8. Tung GA, Davis LM, Wiggins ME, Fadale PD. Tears of the Anterior Cruciate Ligament: Primary and Secondary Signs at MR Imaging. Radiology. 1993;188:661-667.

9. Brandser EA, Riely MA, Berbaum KS, El-Khoury GY, Bennett DL. MR Imaging of Anterior Cruciate Ligament Injury: Independent Value of Primary and Secondary Signs. AJR. 1996;167:121-126.

10. Hong SH, Choi JY, Lee GK, Choi JA, Chung Hw, Kang HS. Grading of ACL injury: Diagnostic efficacy of oblique coronal MRI of the knee. J Cat. 2003;27:5:814-819.

11. Katahira K, Yamashita $Y$, Takahashi M, et al. MR Imaging of the Anterior Cruciate Ligament: Value of thin Slice Direct Oblique coronal Technique. Radiation Medicine. 2001;19:1:1-7.
12. Roychowdhury S, Fitzergerald SW, Sonin AH, Peduto AJ, Miller FH, Hoff FL. Using MR Imaging to Diagnose Partial Tears of the Anterior Cruciate Ligament: Value of Axial Images. AJR. 1997;168:1487-1491.

13. Chen WT, Shih TT, Tu HY, Chen RC, Shau WY. Partial and complete tear of the anterior cruciate ligament. Acta Radiol. 2002;43:5:511-516.

14. Duc SR, Zanettie M, Kramer J, Kach KP, Zollikofer CL, Wentz $\mathrm{KU}$. Magnetic Resonance Imaging of Anterior Cruciate Ligament Tears: Evaluation of Standard Orthogonal and Tailored Paracoronal Images. Acta Radiol. 2005;7:729-733.

15. Lawrence JAL, Ostlere SJ, Dodd CAF. MRI diagnosis of partial tears of the anterior cruciate ligament. Injury. 1996;27: 3:153-155.

16. Umans $\mathrm{H}$, Wimpfhelmer $\mathrm{O}$, Haramati $\mathrm{N}$, Applbaum $\mathrm{YH}$, Adler $\mathrm{M}$, Bosco J. Diagnosis of Partial Tears of the Anterior Cruciate Ligament of the Knee: Value of MR Imaging. AJR. 1995;165: 893-897.

17. Padulo J, Oliva F, Frizziero A, Maffulli N. Muscles, Ligaments and Tendons Journal. Basic principles and recommendations in clinical and field science research: 2016 update. MLTJ. 2016;6 (1):1-5.

18. Bridgman S, Richards PJ, Walley G, et al. MRI does not reduce knee arthroscopy or improve patient outcome for mechanical knee symptoms: A prospective RCT in a UK tertiary centre. Arthroscopy. 2007.

19. McCauley TR, Moses M, Kier R, Lynch JK, Barton JW, JokI P. MR Diagnosis of Tears of Anterior Cruciate Ligament of the Knee: Importance of Ancillary Findings. AJR. 1994;162:115 119.

20. Smith DK, May DA, Phillips P. MR imaging of the anterior cruciate ligament: frequency of discordant findings on sagittaloblique images and correlation with arthroscopic findings. AJR. 1996;166:2:411-413.

21. Vellet AD, Marks P, Fowler P, Munro T. Accuracy of Nonorthogonal Magnetic Resonance Imaging in Acute Disruption of the anterior Cruciate Ligament. Arthroscopy. 1989;5:4: 287-293.

22. Buckwalter KA, Pennes DR. Anterior Cruciate Ligament: Oblique Sagittal MR Imaging. Radiology. 1990;175:276-277.

23. Polly DW Captain, Callaghan JJ, Sikes RA, McCabe JM, McMahon K, Savory CG. The Accuracy of Selective Magnetic Resonance Imaging Compared with the Findings of Arthroscopy of the Knee. J B Joint Surg (Am). 1988;70:192-198.

24. Barry KP, Mesgarzadeh M, Triolo J, Moyer R, Tehranzadeh J, Bonakdarpour A. Accuracy of MRI patterns in evaluating anterior cruciate ligament tears. Skeletal Radiol. 1996;25:4:365370.

25. Hodler J, Haghighi P, Trudell D, Resnick D. The Cruciate Ligaments of the Knee: Correlation Between MR Appearance and Gross and Histologic Findings in Cadaveric Specimens. AJR. 1992;159:357-360.

26. Vahey TN, Broome DR, Kayes KJ, Shelbourne KD. Acute and chronic tears of the anterior cruciate ligament: differential features at MR Imaging. Radiology. 1991;181:1:251-253.

27. Glükert K, Kladny B, Blank-Schäl A, Hofmann G. MRI of the knee joint with a 3-D gradient echo sequence. Equivalent to diagnostic arthroscopy? Arch Orthop Trauma Surg. 1992;112:514.

28. Reeder JD, Matz SO, Becker L, Andelman SM. MR Imaging of the Knee in the Sagittal Projection: Comparison of Three-Dimensional Gradient-Echo and Spin-Echo Sequences. AJR. 1989;153:537-540.

29. Heron CW, Calvert PT. Three-dimensional Gradient-Echo MR Imaging of the Knee: Comparison with Arthroscopy in 100 patients. Radiology. 1992;183:839-844. 
30. Ha TPT, Li KCP, Beaulieu CF, et al. Anterior Cruciate Ligament Injury: Fast Spine-Echo MR Imaging with Arthroscopic Correlation in 217 Examinations. AJR. 1998;170:1215-1219.

31. Linter DM, Kamaric E, Moseley JB, Noble PC. Partial tears of the anterior cruciate ligament. Are they clinically detectable? Am J Sports Med. 1995;23:111.
32. Yao L, Gentilli A, Petrus L, Lee JK. Partial ACL rupture: an MR diagnosis? Skeletal Radiol. 1995;24:247-251.

33. Boric I, Pecina M, Bojanic I, Haspl M, Roic G. Comparison of Conventional Spin-Echo and Fast Spin-Echo Magnetic Resonance Imaging with Fat Suppression in Cruciate Ligament Injury. Croatian Med J. 2004;45:2:195-201. 